Supporting Information

\title{
Study on Crystal Growth Mechanism and Critical Secondary Nucleus Size of Poly(ethylene oxide)/Urea Inclusion Compound
}

Yang Gao, Shujing Zhang, Jiaxin Shi, Baohua Guo, Jun Xu*

Advanced Materials Laboratory of Ministry of Education (MOE),

Department of Chemical Engineering, Tsinghua University, Beijing 100084, China

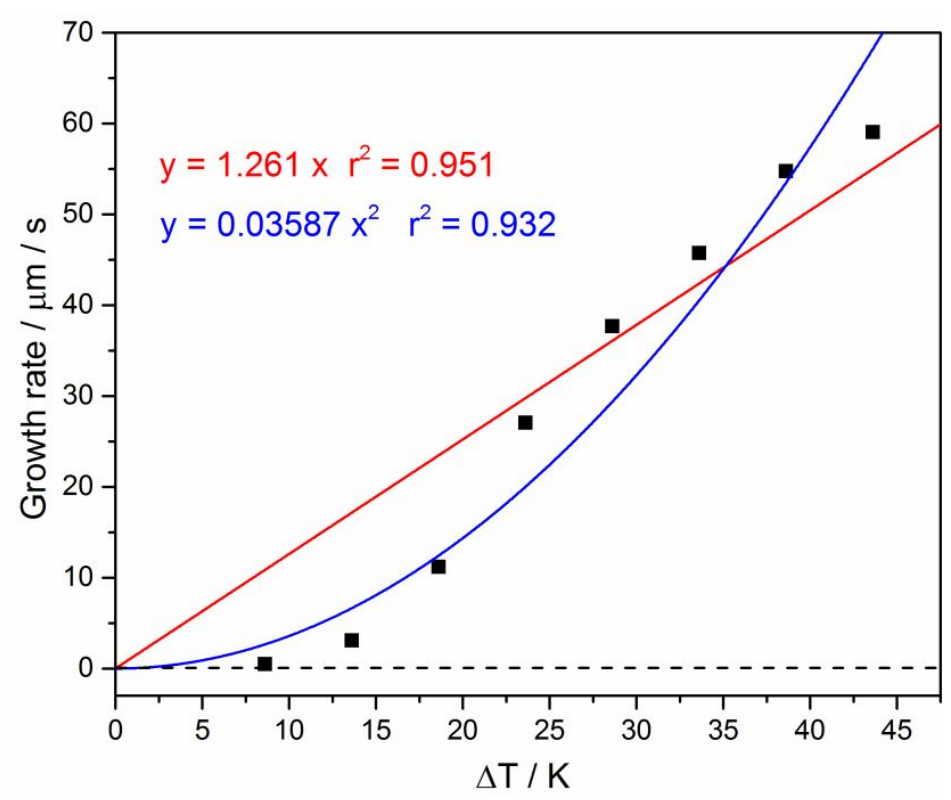

Figure S1. Plot of radial growth rate $G$ versus the degree of supercooling $\Delta T$ at $T_{c}$

from 65 to $100^{\circ} \mathrm{C}$ and its fitting curves for the normal growth mechanism (red curve) and screw dislocation mechanism (blue curve). 


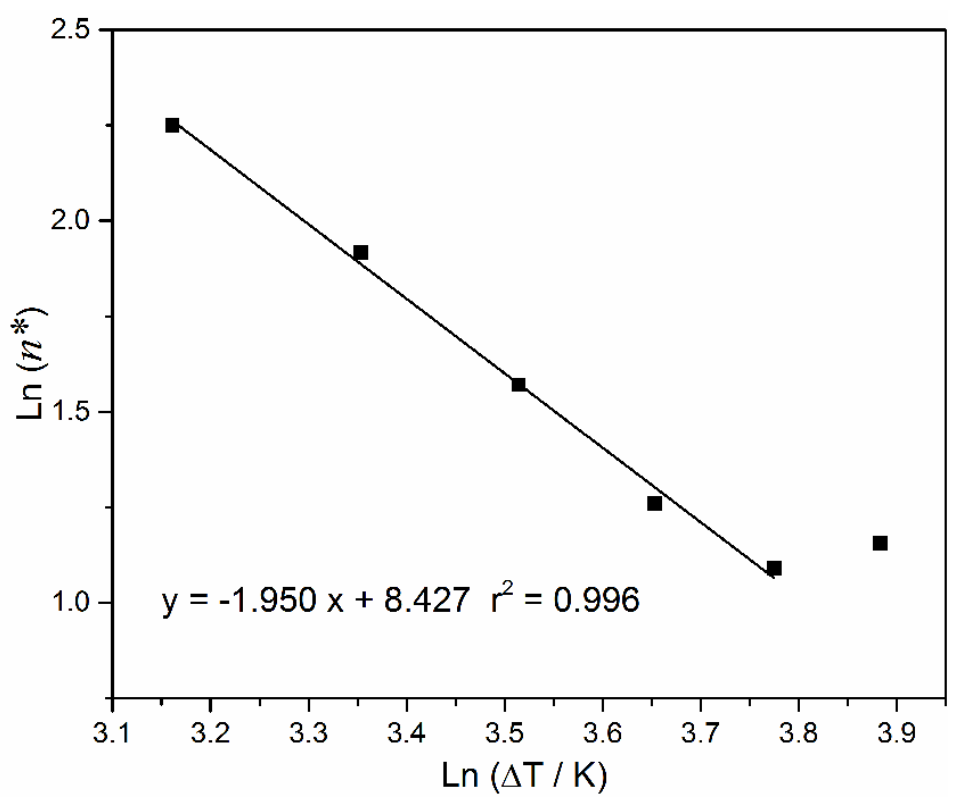

Figure S2. Double logarithmic plot of critical secondary nucleus sizes $n$ * versus the degree of supercooling $\Delta T$ and its linear fitting curve at $T_{c}$ from 65 to $85^{\circ} \mathrm{C}$. The data point at $60{ }^{\circ} \mathrm{C}$ (at the bottom right corner) is not adopted for curve fitting. 